\title{
Correlação entre o equilíbrio funcional e o estadiamento da Doença de Parkinson
}

\author{
Correlation between the functional balance and the staging of Parkinson's Disease \\ Marina Teixeira Pereira', Daniella Meneses Seawright Oliveira', Ana Carolina Alves Maués Dias', \\ Ananda Maria Figueiró de Moraes ${ }^{1}$, George Alberto Silva Dias', Ediléa Monteiro de Oliveira ${ }^{1}$
}

Resumo Objetivo: Determinar se há correlação entre o equilíbrio funcional e o estágio da doença de Parkinson. Método: Foram selecionados 13 pacientes diagnosticados com doença de Parkinson que tiveram a severidade da patologia classificada de acordo com a Escala de Hoehn e Yahr (modificada), utilizada como critério de seleção e avaliação. Em sequência, por meio de questionário, foram coletadas informações pessoais e clínicas. Posteriormente aplicou-se o teste de Tinetti e o teste Timed Up and Go para definição das condições de equilíbrio e marcha. Para a análise das variáveis categóricas utilizaram-se os testes G (Aderência) e o teste de correlação de Spearman $(p \leq 0,05)$. Resultados: No teste de Tinetti determinou-se a presença de 6 pacientes $(46,2 \%)$ com alto risco de queda. Já o TUG apontou que 6 pacientes $(46,2 \%)$ apresentaram alto grau de comprometimento funcional. A Escala de Hoehn e Yahr (modificada) classificou os indivíduos em estágios de leve a moderado. As correlações entre os testes $(\mathrm{r}=-0,8 ; \mathrm{p}=0,0004)$ e entre o Timed Up and Go e Escala de Hoehn e Yahr (modificada) $(\mathrm{r}=0,8 ; \mathrm{p}=0,0001)$ e o teste de Tinetti e mesma escala $(r=-0,8 ; p=0,0006)$ demonstraram correlação forte e significância estatística. Conclusão: Os instrumentos avaliativos do equilíbrio funcional utilizados apresentam correlação com a Escala de Hoehn e Yahr (modificada), auxiliando a determinar a severidade da doença de Parkinson.

Descritores: Doença de Parkinson; equilíbrio postural; marcha.

Summary Purpose: To determine if there is a correlation between functional balance and the stage of Parkinson's disease. Methods: We selected 13 patients diagnosed with Parkinson's disease who had the severity of the pathology classified according to the Hoehn and Yahr Scale (modified), used as selection and evaluation criteria. Subsequently, through a questionnaire, personal and clinical information were collected. Afterwards the Tinetti test and the Timed Up and Go test were applied to define the conditions of balance and gait. For the analysis of the categorical variables, the G (Adherence) tests and the Spearman correlation test were used $(p \leq 0,05)$. Results: In Tinetti's test we determined the presence of 6 patients $(46.2 \%)$ with a high risk of falls. The TUG indicated that 6 patients $(46.2 \%)$ had a high degree of functional impairment. The Hoehn and Yahr Scale (modified) ranked individuals in mild to moderate stages. The correlations between the tests $(r=-0.8, p=0.0004)$ and between Timed Up and Go and Hoehn and Yahr Scale (modified) $(r=0.8, p=0.0001)$ and Tinetti's test and the same scale $(r=-0.8 ; p=0.0006)$ demonstrated strong correlation and statistical significance. Conclusion: The functional balance evaluation instruments used correlate with the Hoehn and Yahr Scale (modified) helping to determine the severity of Parkinson's disease.

Keywords: Parkinson Disease; postural balance; gait.

'Universidade do Estado do Pará - UEPA, Belém, PA, Brasil

Fonte de financiamento: Nenhuma.

Conflito de interesses: Os autores declaram não haver conflitos de interesse.

Recebido: Julho 03, 2018

Aceito: Julho 12, 2018

Trabalho realizado na Universidade do Estado do Pará - UEPA e na Universidade da Amazônia - UNAMA, Belém, PA, Brasil. 


\section{Introdução}

A doença de Parkinson (DP) é uma doença crônica degenerativa de caráter progressivo que acomete o sistema nervoso central (SNC), caracterizando-se por uma alteração no controle motor em decorrência da degeneração de neurônios dopaminérgicos presentes na substância negra do mesencéfalo ${ }^{1,2}$.

Apresenta a segunda maior prevalência dentre as patologias neurodegenerativas, ficando atrás apenas da doença de Alzheimer ${ }^{3}$. Dentre as diversas alterações presentes na DP, a instabilidade postural é apontada como uma das mais características ${ }^{4}$. A instabilidade postural, implícita no deficiente controle de equilíbrio estático e dinâmico, se expressa na dificuldade encontrada por esses pacientes em gerar respostas às perturbações posturais ${ }^{5}$.

Conforme a doença evolui, maior a dificuldade de o paciente manter o equilíbrio funcional ${ }^{6}$. Corriqueiramente, a progressão sintomática da doença é definida através dos estágios da Escala de Hoehn e Yahr, que utiliza números de 1 a 5 para classificar a severidade da patologia. Essa ferramenta é demarcada pelo agravamento das condições de marcha e pela instabilidade postural ${ }^{7}$. Os pacientes com DP acumulam ao longo do desenvolvimento da doença inúmeras alterações posturais que somadas aos demais distúrbios comprometem a orientação postural ${ }^{4}$.

A Escala de Hoehn e Yahr é o instrumento mais adequado para a determinação do grau de incapacidade desses pacientes, fornecendo uma visão global do estado em que se encontram ${ }^{8}$. Entretanto, torna-se necessária a busca por novos instrumentos objetivos de avaliação da progressão da DP que impulsionem a constante modificação e adequação das terapêuticas disponíveis ${ }^{9}$. Como a marcha e o equilíbrio são caracteristicamente afetados com o curso da doença os mesmos poderiam relacionar-se a sua gravidade.

Nesse sentido, a aplicação de testes simples como o Timed Up and Go (TUG) e o Teste de Tinetti, já validados para essa população, podem contribuir para uma melhor compreensão das condições de equilíbrio funcional, características associadas ao grau de incapacidade ${ }^{9-11}$. Além de fornecerem resultados confiáveis, oferecem menor custo e menor tempo de execução quando comparados a alternativas mais tecnológicas .

Mediante os dados expostos anteriormente, o presente estudo visou, por meio da aplicação de testes, avaliar se há correlação entre o equilíbrio funcional e o estadiamento da doença de Parkinson de pacientes atendidos em duas unidades de ensino e assistência em Fisioterapia.

\section{Metodologia}

Trata-se de um estudo de campo, observacional, transversal, de caráter quantitativo. A pesquisa teve início após a aprovação da mesma pelo Comitê de Ética em Pesquisa com Seres Humanos (parecer n: 1.095.711).

Participaram do estudo pacientes de ambos os sexos portadores de doença de Parkinson em atendimento em duas clínicas escola de Fisioterapia, situadas na cidade de Belém, Pará; com idade a partir de 40 anos; que preenchessem os critérios clínicos de doença de Parkinson idiopática e fossem classificados nos estágios de 1 a 3 da patologia na Escala de Hoehn e Yahr (modificada); além de assinar o Termo de Consentimento Livre e Esclarecido (TCLE). A amostra foi composta por indivíduos recrutados por demanda espontânea após a divulgação do estudo.

Foram excluídos aqueles que apresentassem comorbidades limitantes à aplicação dos instrumentos avaliativos ou possuíssem grau de demência determinado pelo Mini Exame do Estado Mental (MEEM).

Primeiramente, os participantes foram submetidos à aplicação do MEEM. Nesta pesquisa consideraram-se as seguintes notas de corte de acordo com a escolaridade: $<18$ para analfabetos, $<21$ para indivíduos com 1 a 4 anos de estudo, $<24$ para 5 a 8 anos e $<26$ para aqueles com nível educacional acima de 8 anos $^{12}$.

Em sequência, para seleção e avaliação dos pacientes utilizou-se a Escala de Hoehn e Yahr (modificada). Esta escala possui oito estágios de classificação que variam do estágio 0 , que corresponde a nenhum sinal da doença; estágio 1, doença unilateral; estágio 1,5, no qual há envolvimento unilateral e axial; estágio 2, evolução da doença com acometimento bilateral porém sem déficit de equilíbrio; estágio 2,5, doença bilateral leve, com recuperação no "teste do empurrão"; estágio 3, doença bilateral leve a moderada, alguma instabilidade postural, capacidade para viver independente; estágio 4, incapacidade grave, incapaz de caminhar ou permanecer de pé sem auxílio, ao estágio 5, confinado à cama ou cadeira de rodas, a não ser que receba ajuda ${ }^{13}$.

Com o intuito de coletar informações sociodemográficas e características clínicas dos pacientes, aplicou-se um formulário para coleta de dados especificamente desenvolvido pelos pesquisadores para utilização nesse estudo. $\mathrm{O}$ formulário reúne questões acerca de informações pessoais, tempo de diagnóstico da DP, uso de medicação contínua, ocorrência de quedas nos últimos doze meses e o quantitativo das mesmas. 
Posteriormente, os indivíduos executaram os testes de equilíbrio funcional e mobilidade. O teste de Tinetti, considerado altamente confiável, mensura o equilíbrio e a marcha tendo sua aplicabilidade validada para populações idosas e com patologias especificas, como a $\mathrm{DP}^{7,14}$. Possui 16 itens divididos em dois grupos: teste de equilíbrio (16 pontos) e teste de marcha (12 pontos $)^{14}$.

Por fim, utilizou-se o TUG, teste que mensura o tempo que um indivíduo despende para levantar de uma cadeira sem braços, caminhar uma distância de três metros, realizar um giro de $180^{\circ}$, retornar e sentar na mesma cadeira ${ }^{15}$. Tem por finalidade analisar a mobilidade funcional e o equilíbrio, além de indicar o risco de quedas ${ }^{16}$.

Para auxiliar na aplicação dos testes foram utilizados uma fita métrica simples de $1 \mathrm{~m}$, uma cadeira fixa secretária simples sem braço e um cronômetro para monitorização do tempo de execução do participante. A análise descritiva foi realizada pelo software EpiInfo ${ }^{\circledR}$ 3.5.2. Já, para a análise das variáveis categóricas utilizaram-se os testes $\mathrm{G}$ (Aderência) e o teste de correlação de Spearman, através do software BioEstat ${ }^{\circledR} 5.0$ e para a confecção dos gráficos, o software GraphPad Prism ${ }^{\circledR} 5.0$. Adotou-se valor de p de 0,05 (5\%).

\section{Resultados}

O estudo contou com uma amostra de 13 voluntários com diagnóstico de DP. Os dados referentes à caracterização da amostra estão dispostos na Tabela 1. Com relação ao sexo dos participantes, observou-se predominância do sexo feminino. Quanto à idade, verificaram-se extremos de $48-83$ anos. Os indivíduos foram questionados ainda sobre a utilização de medicação contínua. Todos afirmaram fazer uso de medicamentos para tratamento da doença de Parkinson, e do total de avaliados, apenas 1 relatou não fazer uso de Levodopa, droga mais utilizada no tratamento dessa patologia.

Tabela 1. Dados para caracterização da amostra

\begin{tabular}{|c|c|c|c|}
\hline Variáveis & n & $\%$ & IC $95 \%$ \\
\hline \multicolumn{4}{|l|}{ Sexo $(n=13)$} \\
\hline Masculino & 6 & 46 & $19,2-74,9$ \\
\hline Feminino & 7 & 54 & $25,1-80,8$ \\
\hline \multicolumn{4}{|l|}{ Idade $(n=13)$} \\
\hline Média \pm Desvio Padrão & \multicolumn{2}{|c|}{$63,6 \pm 13,7$ anos } & $55,8-70,7$ \\
\hline Mediana & \multicolumn{2}{|c|}{65 anos } & \\
\hline \multicolumn{4}{|l|}{ Tempo de diagnóstico $(n=13)$} \\
\hline Média \pm Desvio Padrão & \multicolumn{2}{|c|}{$4,84 \pm 5,1$ anos } & $2,0-7,6$ \\
\hline Mediana & \multicolumn{2}{|c|}{4 anos } & \\
\hline \multicolumn{4}{|l|}{ Estado civil $(\mathrm{n}=13)$} \\
\hline Solteiro & 1 & 7,7 & $0,2-36,0$ \\
\hline Casado & 7 & 53,8 & $25,1-80,8$ \\
\hline Divorciado & 3 & 23,1 & $5,0-53,8$ \\
\hline Viúvo & 2 & 15,4 & $1,9-45,4$ \\
\hline \multicolumn{4}{|l|}{ Escolaridade $(\mathrm{n}=13)$} \\
\hline Fundamental Incompleto & 3 & 23,1 & $5,0-53,8$ \\
\hline Fundamental Completo & 0 & 0 & $100-100$ \\
\hline Ensino Médio Incompleto & 4 & 30,8 & $9,1-61,4$ \\
\hline Ensino Médio Completo & 2 & 15,4 & $1,9-45,4$ \\
\hline Superior Incompleto & 0 & 0 & $100-100$ \\
\hline Superior Completo & 4 & 30,8 & $9,1-61,4$ \\
\hline \multicolumn{4}{|l|}{ Ocupação $(n=13)$} \\
\hline Dona de Casa & 1 & 7,7 & $0,2-36,0$ \\
\hline Pensionista & 5 & 38,5 & $13,9-68,4$ \\
\hline Aposentado & 5 & 38,5 & $13,9-68,4$ \\
\hline Aposentado + trabalho & 2 & 15,4 & $19-45,4$ \\
\hline
\end{tabular}

$*(p \leq 0,05)$. 
A Tabela 2 apresenta os resultados do teste de Tinetti, TUG e da aplicação da Escala de Hoehn e Yahr (modificada). No teste de Tinetti, dos $6(46,2 \%)$ pacientes classificados com alto risco de queda, 4 relataram ter sofrido pelo menos uma queda nos últimos 12 meses. Além disso, desse total, 2 estavam no estágio 2,5 e 4 no estágio 3 da Escala de Hoehn e Yahr (modificada). Já o TUG apontou que 6 pacientes (46,2\%) apresentaram alto grau de comprometimento funcional, $3(23 \%)$ mobilidade considerada normal para idosos com fragilidade ou adultos com alguma patologia limitante e $4(30,8 \%)$ foram considerados com mobilidade normal. Dos 6 parkinsonianos com maior tempo de execução do TUG, 3 relataram pelo menos um episódio de queda nos últimos 12 meses. A Escala de Hoehn e Yahr (modificada) identificou os pacientes avaliados como portadores de incapacidade leve a moderada.

Efetuou-se a correlação entre os dois testes de equilíbrio funcional, teste de Tinetti e TUG, obtendo-se forte correlação negativa( $(r=-0,8)$ com resultados estatisticamente significantes $(p=0,0004)$, o que indica que quanto maior o tempo que o indivíduo leva para executar a tarefa proposta pelo TUG, maior o risco de queda, fator determinado pelo teste de Tinetti (Figura 1).

Observaram-se resultados semelhantes nas duas outras correlações realizadas. A correlação entre a Escala de Hoehn e Yahr (modificada) e o teste de Tinetti demonstrou-se forte e negativa $(\mathrm{r}=-0,8)$, determinando que quanto mais avançado o estágio da DP, maior o risco de ocorrência de quedas (Figura 2). Por fim, obteve-se forte correlação positiva $(r=0,8)$ entre a Escala de Hoehn e Yahr (modificada) e o TUG, indicando que quanto maior a classificação da severidade da doença, maior é o tempo despendido pelo indivíduo para a realização do teste (Figura 3).

Tabela 2. Valores obtidos no teste de Tinetti, Timed Up and Go (TUG) e Escala de Hoehn e Yahr (modificada) (HY)

\begin{tabular}{llll}
\hline \multicolumn{1}{c}{ Variáveis } & $\mathbf{n}$ & $\mathbf{\%}$ & IC $\mathbf{9 5 \%}$ \\
\hline Tinetti (n=13) & & & $19,2-74,9$ \\
\hline Alto risco de queda & 6 & 46,2 & $13,9-68,4$ \\
\hline Médio risco de queda & 5 & 38,5 & $1,9-45,4$ \\
\hline Baixo risco de queda & 2 & 15,4 & $5,0-53,8$ \\
\hline TUG (n=13) & 3 & & $9,1-61,4$ \\
\hline Normal & 4 & 23,1 & $5,0-53,8$ \\
\hline Boa mobilidade & 3 & 30,8 & $5,0-53,8$ \\
\hline Regular mobilidade & 3 & 23,1 & 23,1 \\
\hline Mobilidade prejudicada & & & $5,0-53,8$ \\
\hline HY (n=13) & 3 & 23,1 & $1,9-45,4$ \\
\hline Estágio 1 & 2 & 15,4 & $9,1-61,4$ \\
\hline Estágio 1,5 & 4 & 30,8 & $9,1-61,4$ \\
\hline Estágio 2,5 & 4 & 30,8 & \\
\hline Estágio 3 & & & \\
\hline
\end{tabular}

$*(\mathrm{p} \leq 0,05)$.

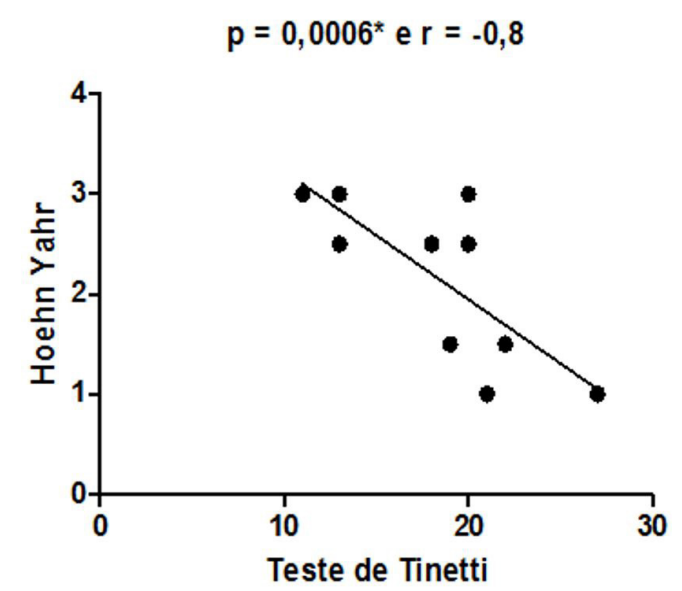

Figura 1. Correlação entre o teste de Tinetti e o TUG. 


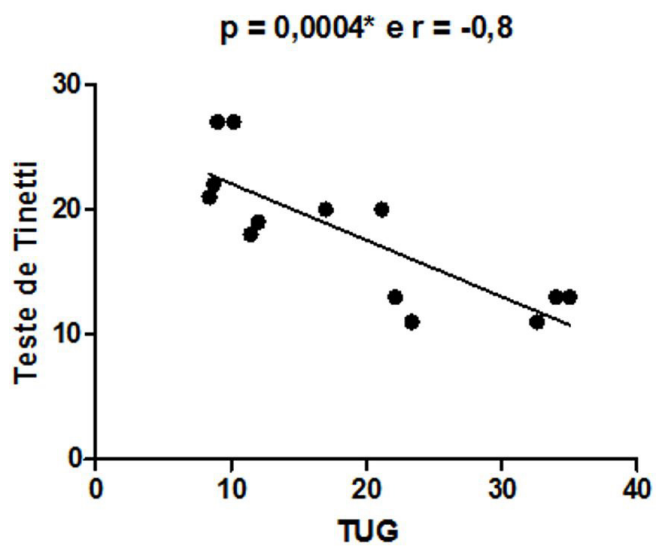

Figura 2. Correlação entre a Escala de Hoenh e Yahr (modificada) e o teste de Tinetti.

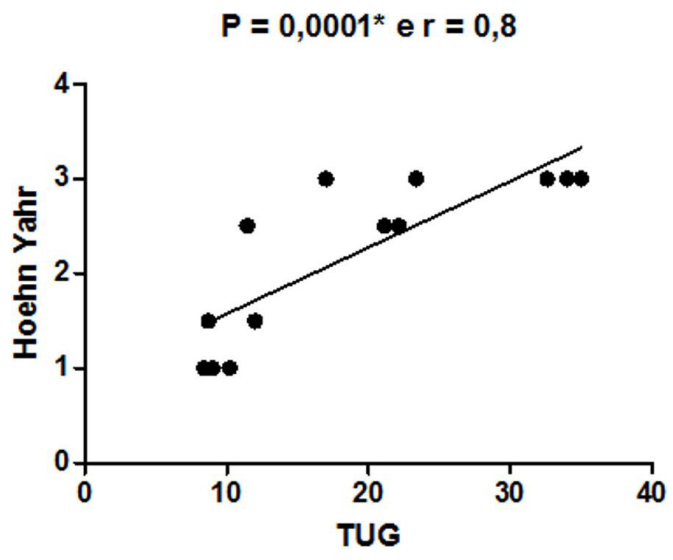

Figura 3. Correlação entre a Escala de Hoehn e Yahr (modificada) e o TUG.

\section{Discussão}

Na presente pesquisa, a correlação efetuada entre o teste de Tinetti e o TUG mostrou-se forte e negativa $(r=-0,8)$ e com resultados significantes. Estes achados corroboram com os de estudo com grupo de idosos submetidos a exercícios físicos o qual obteve uma correlação negativa entre o equilíbrio $(\mathrm{r}=-0,335)$ avaliado pelo teste de Tinetti e o seu valor total $(\mathrm{r}=-0,385)$ com Timed Up and $\mathrm{Go}(\mathrm{p}=0,001)^{17}$. Outra pesquisa, realizada com pacientes oncogeriátricos em ambiente hospitalar alcançou resultados semelhantes ao correlacionar estes dois testes $(r=-0,952)^{18}$. Isto demonstra que quanto maior é a pontuação do teste de Tinetti menor é tempo no teste TUG.

No entanto, esses dados confrontam aqueles obtidos por Karuka et al. ${ }^{19}$ que ao analisarem a Escala de Tinetti (Performance Oriented Mobility Assessment-POMA), versão expandida do teste de Tinetti, e o TUG não encontraram correlação entre esses dois instrumentos avaliativos $(r=-0,21)$, em pesquisa realizada com idosos hígidos. A aplicação tanto da Escala de Tinetti, quanto do teste de Tinetti, é mais bem indicada em indivíduos com maior grau de incapacidade e maior risco de quedas. Esse fato deve ser considerado ao se analisar essa divergência.

Os resultados deste estudo demonstraram a relação existente entre a Escala de Hoehn e Yahr (modificada) e o teste de Tinetti e o Timed Up and Go. Esse fato determina que uma pior classificação nos estágios da doença de Parkinson influencia na detecção de piores condições de equilíbrio funcional.

Os resultados do estudo demonstraram forte correlação negativa e com significância estatística entre a severidade da doença, determinada pela Escala de Hoehn e Yahr (modificada) e o desempenho no teste de Tinetti $(r=-0,8)$, evidenciando a clara influência da progressão da doença na deterioração do equilíbrio e consequente aumento no risco de quedas. Em estudo de Kegelmeyer, Kloos, Thomas e Kostyk ${ }^{7}$ que buscou determinar a confiabilidade e validade do teste de Tinetti para indivíduos com DP, obteve-se correlação 
igualmente negativa ( $\mathrm{rs}=-45, \mathrm{P}<0,05$.) entre o teste de Tinetti e outro instrumento indicador da evolução patológica, a Escala Unificada de Avaliação da Doença de Parkinson (UPDRS).

A correlação efetuada no presente estudo entre a Escala de Hoehn e Yahr (modificada) e o teste Timed Up and Go, assim como as demais correlações, demonstrou-se forte e com significância estatística $(\mathrm{r}=0,8)$. Resultado similar foi observado por Pelicioni et al. ${ }^{20}$ em pesquisa que dividiu parkinsonianos em dois grupos: tremor dominante (TD) e acinesia (AR). Em cada grupo, obteve-se correlação positiva e significante (TD: 0,46 / AR: 0,68). Em outro estudo que aplicou três diferentes testes para mensurar a mobilidade funcional de indivíduos com DP, verificou-se também correlação, porém moderada entre a versão original da Escala de Hoehn e Yahr e o TUG e $(r=0,54)^{21}$.

A aplicação do teste de Tinetti gerou uma resposta preocupante, 84,7\% dos voluntários deste estudo já possuem de médio a alto risco de quedas, tendo um número expressivo relatado ser caidor. O decréscimo das condições de equilíbrio é um dos sintomas mais frequentes em indivíduos com $\mathrm{DP}^{22}$. Esse déficit é um dos principais responsáveis pela ocorrência de quedas, complicação comum e incapacitante que progride com o avançar da doença ${ }^{23}$. Em estudo de Allen et al. ${ }^{24}$, de 35 a $90 \%$ dos pacientes relataram ter sofrido pelo menos uma queda por ano e dois terços destes, quedas recorrentes.

Ao analisar os tempos obtidos na execução do TUG, obteve-se média de 18,6 segundos. De acordo com a classificação do teste, percebe-se que em grande parte dos parkinsonianos $(46,2 \%)$ foi detectado alto grau de comprometimento funcional. Um pior desempenho no TUG tem íntima relação com uma menor velocidade de marcha ${ }^{25}$. Inúmeros estudos comparativos entre populações saudáveis e com doença de Parkinson deixam clara a diferença entre as mesmas, ressaltando a pior mobilidade de portadores de $\mathrm{DP}^{11,26}$.

De acordo com o estadiamento da doença feito pela Escala de Hoehn e Yahr (modificada), percebe-se que 8 dos 13 parkinsonianos estão situados em estágios intermediários da patologia (2,5 - 3), sendo a mediana do tempo de diagnóstico de todos os participantes referente a 4 anos. Isso demonstra que, apesar do conhecido caráter progressivo da DP, não há relação direta entre seu tempo de manifestação e subsequente diagnóstico, com a classificação em um estágio mais severo na referida escala. Um estudo de coorte reforça esse achado, ao analisar uma mesma população de portadores de doença de Parkinson, que não fazia uso de medicação específica para o tratamento, por um período de 10 anos. Dos 15 pacientes, 9 mantiveram-se nos mesmos estágios ${ }^{27}$.

Apesar da amostra reduzida, observa-se que os resultados deste estudo, além de explicitar a relação existente entre a deterioração ocasionada pelo avanço da doença e o agravo nas condições de equilíbrio e mobilidade, devem impulsionar a elaboração de novos trabalhos científicos.

\section{Conclusão}

Diante dos resultados obtidos, pode-se concluir que instrumentos avaliativos do equilíbrio funcional, especificamente, o teste de Tinetti e o Timed Up and Go apresentam correlação com a Escala de Hoehn e Yahr (modificada), auxiliando-a não só na avaliação das condições de equilíbrio e mobilidade, como na determinação da progressão da doença de Parkinson.

\section{Referências}

1. Silva DM, Nunes COM, Oliveira PJAL, Coriolano MGWS, Berenguer FA, Lins OG, et al. Efeitos da fisioterapia aquática na qualidade de vida de sujeitos com doença de Parkinson. Fisioter Pesqui. 2013;20(1):17-23. http://dx.doi.org/10.1590/S1809-29502013000100004.

2. Barbieri FA, Batistela RA, Rinaldi NM, Teixeira-Arroyo C, Stella F, Gobbi LTB. Efeito do exercício físico na amplitude de movimento articular dos membros inferiores de indivíduos com doença de Parkinson. Fisioter Pesqui. 2014;21(2):167-73. http://dx.doi.org/10.1590/1809-2950/50421022014.

3. Torrent R, De Angelis Rigotti F, Dell'Era P, Memo M, Raya A, Consiglio A. Using iPS Cells toward the Understanding of Parkinson's Disease. J Clin Med. 2015;4(4):548-66. http://dx.doi.org/10.3390/jcm4040548. PMid:26239346.

4. Takeuti T, Maki T, Silva CVR, Soares AJ, Duarte J. Correlação entre equilíbrio e incidência de quedas em pacientes portadores de doença de Parkinson. Rev Neurocienc. 2011;19(2):237-43.

5. Rinalduzzi S, Trompetto C, Marinelli L, Alibardi A, Missori P, Fattapposta F, et al. Balance dysfunction in Parkinon's Disease. BioMed Res Int. 2015;2015:434683. http://dx.doi.org/10.1155/2015/434683. PMid:25654100. 
6. Harris DM, Rantalainen T, Muthalib M, Johnson L, Teo W-P. Exergaming as a viable therapeutic tool to improve static and dynamic balance among older adults and people with idiopathic Parkinson's disease: a systematic review and meta-analysis. Front Aging Neurosci. $2015 ; 7: 167$. http://dx.doi.org/10.3389/fnagi.2015.00167. PMid:26441634.

7. Kegelmeyer DA, Kloos AD, Thomas KM, Kostyk SK. Reliability and validity of the tinetti mobility test for individuals with Parkinson disease. Phys Ther. 2007;87(10):1369-78. http://dx.doi.org/10.2522/ptj.20070007. PMid:17684089.

8. Ramos ML, Neves DR, Lima VP, Orsini M, Machado D, Bastos VHV, et al. Análise de parâmetros pneumofuncionais em pacientes com doença de Parkinson: estudo piloto. Rev Bras Neurol. 2014;50(2):38-43.

9. Dewey DC, Miocinovic S, Bernstein I, Khemani P, Dewey III RB, Querry R, et al. Automated gait and balance parameters diagnose and correlate with severity in Parkinson disease. J Neurol Sci. 2014;345(1-2):131-8. http://dx.doi.org/10.1016/j.jns.2014.07.026. PMid:25082782.

10. Horak FB, Mancini M. Objective biomarkers of balance and gait for Parkinson's disease using body-worn sensors. Mov Disord. 2013;28(11):154451. http://dx.doi.org/10.1002/mds.25684. PMid:24132842.

11. Xu D, Cole MH, Mengersen K, Silburn PA, Qiu F, Graepel C, et al. Executive function and postural instability in people with Parkinson's disease. Parkinsons Dis. 2014;2014: 684758. http://dx.doi.org/10.1155/2014/684758. PMid:25136474.

12. Ikuta YM, Reis CRM, Ramos $M M A B$, Borges $M M G$, Araújo MC. Avaliação da função cognitiva em pacientes com doença de Parkinson. Rev Para Med. 2012;26(1):1-6.

13. Pinheiro IM, Santos LLS, Paula LCN, Costa ACN. Impacto da Doença de Parkinson na funcionalidade e qualidade de vida de idosos em uma unidade de referência geriátrica na cidade de Salvador - Bahia. Rev Ciênc Méd Biol. 2014;13(3):292-7. http://dx.doi.org/10.9771/cmbio. v13i3.12933.

14. King LA, Mancini M, Priest K, Salarian A, Rodrigues-de-Paula F, Horak F. Do clinical scales of balance reflect turning abnormalities in people with Parkinson's disease? J Neurol Phys Ther. 2012;36(1):25-31. http://dx.doi.org/10.1097/NPT.0b013e31824620d1. PMid:22333919.

15. Almeida ST, Soldera CL, Carli GA, Gomes I, Resende TL. Análise de fatores extrínsecos e intrínsecos que predispõem a quedas em idosos. Rev Assoc Med Bras. 2012;58(4):427-33. http://dx.doi.org/10.1590/S0104-42302012000400012. PMid:22930020.

16. Cândido DP, Cillo BAL, Fernandes AS, Nalesso RP, Jakaitis F, Santos DG. Análise dos efeitos da dupla tarefa na marcha de pacientes com Doença de Parkinson: relato de três casos. Rev Neurocienc. 2012;20(2):240-5.

17. Ma L, Qiao Y-L, Zhang L, Liu Y, Fan J-H, Gao X. The natural progression of Parkinson's disease in a small cohort with 15 drug-naïve patients. Chin Med J (Engl). 2015;128(13):1761-4. http://dx.doi.org/10.4103/0366-6999.159350. PMid:26112717.

18. Silva A, Almeida GJM, Cassilhas RC, Cohen M, Peccin MS, Tufik S, et al. Equilíbrio, coordenação e agilidade deidosos submetidos à prática de exercíciosfísicos resistidos. Rev Bras Med Esporte. 2008;14(2):88-93. http://dx.doi.org/10.1590/S1517-86922008000200001.

19. Pereira EEB, Santos NB, Sarges ESNF. Avaliação da capacidade funcional do paciente oncogeriátrico hospitalizado. Rev Pan-Amaz Saude. 2014;5(4):37-44. http://dx.doi.org/10.5123/S2176-62232014000400005.

20. Karuka AH, Silva JAMG, Navega MT. Análise da concordância de instrumentos de avaliação do equilíbrio corporal em idosos. Rev Bras Fisioter. 2011;15(6):460-6. http://dx.doi.org/10.1590/S1413-35552011000600006. PMid:22218711.

21. Pelicioni PHS, Pereira MP, Lahr J, Gobbi LTB. Análise cinética e cinemática do levantar e andar em jovens e idosos. Rev Bras Ciênc Esporte. 2015;37(3):237-44. http://dx.doi.org/10.1016/j.rbce.2013.05.002.

22. Verheyden G, Kampshoff CS, Burnett ME, Cashell J, Martinelli L, Nicholas A, et al. Psychometric properties of 3 functional mobility tests for people with Parkinson disease. Phys Ther. 2014;94(2):230-9. http://dx.doi.org/10.2522/ptj.20130141. PMid:24231225.

23. Christofoletti G, Freitas RT, Cândido ER, Cardoso CS. Eficácia de tratamento fisioterapêutico no equilíbrio estático e dinâmico de pacientes com doença de Parkinson. Fisioter Pesqui. 2010;17(3):259-63. http://dx.doi.org/10.1590/S1809-29502010000300013.

24. Hoskovcová $M$, Dušek P, Sieger T, Brožová $H$, Zárubová $K$, Bezdíček $O$, et al. Predicting falls in Parkinson disease: what is the value of instrumented testing in off medication state? PLoS One. 2015;10(10): e0139849. http://dx.doi.org/10.1371/journal.pone.0139849. PMid:26443998.

25. Allen NE, Schwarzel AK, Canning CG. Recurrent falls in Parkinson's disease: a systematic review. Parkinsons Dis. 2013;2013:906274. http:// dx.doi.org/10.1155/2013/906274. PMid:23533953.

26. Cruz SP, Luengo AVG, Lambeck J. Effects of an Ai Chi fall prevention programme for patients with Parkinson's disease. Neurologia. 2016;31(3):176-82. PMid:26372407.

27. Tremblay LE, Esculier JF, Vaudrin J, Bériault P, Gagnon K. Home-based balance training programme using wii fit with balance board for Parkinson's disease: a pilot study. J Rehabil Med. 2012;44(2):144-50. http://dx.doi.org/10.2340/16501977-0922. PMid:22266676. 


\section{Autor correspondente}

Ediléa Monteiro de Oliveira

Travessa Perebebuí, 2623, Marco

CEP 66087-662, Belém, PA, Brasil

Tel.: (91)3131-1704

E-mail: edileaoliveira@yahoo.com.br

Informação sobre os autores

MTP, DMSO, ACAMD e AMFM são bacharéis em Fisioterapia pela Universidade do Estado do Pará (UEPA).

GASD é docente do curso de Fisioterapia da Universidade do Estado do Pará (UEPA); bacharel em Fisioterapia pela Universidade do Estado do Pará (UEPA); doutor em Doenças Tropicais pela Universidade Federal do Pará (UFPA)

EMO é docente do curso de Fisioterapia da Universidade do Estado do Pará (UEPA); bacharel em Fisioterapia pela Universidade do Estado do Pará (UEPA); mestre em Ciência da Motricidade Humana pela Universidade Castelo Branco (UCB-RJ).

\section{Contribuição dos autores}

Todos os autores contribuíram igualmente para a realização do estudo em todas as suas etapas.

Todos os autores leram e aprovaram a versão final submetida ao Pará Research Medical Journal. 